\title{
Characterization of an Outbreak of Extended-Spectrum $\beta$-Lactamase-Producing Klebsiella pneumoniae in a Neonatal Intensive Care Unit in Italy
}

\author{
Marta Corbella, ${ }^{1,2, *}$ Mariasofia Caltagirone, ${ }^{3, *}$ Stefano Gaiarsa,,4 Bianca Mariani,, Davide Sassera, \\ Ibrahim Bitar, Alba Muzzi, Roberta Migliavacca, ${ }^{3}$ Luigia Scudeller, Mauro Stronati, and Patrizia Cambieri ${ }^{1}$
}

Here we report an outbreak of extended spectrum $\beta$-lactamase-producing Klebsiella pneumoniae that occurred in a neonatal intensive care unit in Northern Italy and involved 97 patients. Progressively tightened sets of containment measures were implemented but the epidemic event was stopped only 9 months later. The final, effective, containment strategy consisted of the application of strict geographic cohorting of colonized infants and their nursing staff, the suspension of any new admission and a rigorous daily sterilization protocol for all surfaces and fomites in the ward. A posteriori characterization of the outbreak strain was performed using both traditional microbiology and molecular biology techniques, and whole genome sequencing, allowing to compare outbreak isolates with other strains collected in the previous two years. The results allowed to determine that the outbreak strain had been circulating inside the ward since the year before. Genomic characterization revealed that the strain carried a wide array of virulence and antibiotic resistance determinants, including gene

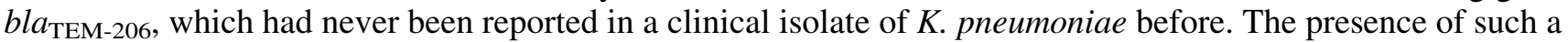
high number of determinants for antibiotic resistance imposes significant therapeutic limitations on the treatment of infections, thus, further epidemiological investigations are needed to evaluate the prevalence of the newly described variant.

Keywords: TEM-206, neonatal intensive care unit, Klebsiella pneumoniae, ESBL, geographic cohorting

\section{Introduction}

$\mathbf{E}$ NTEROBACTERIACEAE REPRESENT A MAJOR cause of nosocomial infections. Chemotherapeutic options available to clinicians for treatment of invasive Enterobacteriaceae infections have been reduced by the rapid emergence and spread of antimicrobial-resistant variants. Of particular concern is the ability of these bacilli to accumulate co-resistance and cross-resistance mechanisms, which has culminated in the circulation of multidrug-resistant (MDR) strains. ${ }^{1}$ Among the causes indicated for the dissemination of MDR Gramnegatives there are overuse of antimicrobial drugs, with a prominent role of third generation cephalosporins, cross- transmission caused by the hands of nurses and other hospital personnel, and failure of infection control practices.

These issues are particularly pronounced in Neonatal Intensive Care Units (NICUs), where the admitted newborns are exposed to a high risk of nosocomial infections development, ${ }^{2}$ due to the severity of their illness and to the exposure to invasive medical devices (e.g., mechanical ventilation, central venous catheter). ${ }^{3}$

Among Gram-negative MDR pathogens, Klebsiella pneumoniae $(\mathrm{Kp})$ is one of the most common and dangerous causes of nosocomial infections, especially in NICUs. Kp shows a clear trend to spread clonally within healthcare institutions and to cause nosocomial outbreaks thanks to MDR

\footnotetext{
${ }^{1}$ U.O.C. Microbiologia e Virologia, Fondazione IRCCS Policlinico San Matteo, Pavia, Italy.

${ }^{2}$ Servizio Biometria ed Epidemiologia Clinica, Direzione Scientifica, Fondazione IRCCS Policlinico San Matteo, Pavia, Italy.

${ }^{3}$ Dipartimento di Scienze Clinico-Chirurgiche Diagnostiche e Pediatriche, Unità di Microbiologia e Microbiologia Clinica, Università di Pavia, Pavia, Italy.

${ }_{5}^{4}$ Dipartimento di Bioscienze, Università degli Studi di Milano, Milano, Italy.

${ }^{5}$ Dipartimento di Biologia e Biotecnologie "L. Spallanzani," Università degli Studi di Pavia, Pavia, Italy.

${ }^{6}$ U.O.C. Direzione Medica di Presidio, Fondazione IRCCS Policlinico San Matteo, Pavia, Italy.

${ }^{7}$ U.O.C. Neonatologia e Terapia Intensiva Neonatale, Fondazione IRCCS Policlinico San Matteo, Pavia, Italy.

*These authors' contributed equally to this work.
} 
phenotypes. ${ }^{4}$ Indeed, most of the Kp clones that are responsible for outbreaks in neonatal wards exhibit resistance to penicillins, broad-spectrum cephalosporins, and monobactams through the production of extended spectrum $\beta$-lactamase (ES $\beta \mathrm{L}){ }^{5}$

In Europe, individual cases or outbreaks of ES $\beta$ L-producing $\mathrm{Kp}(\mathrm{ES} \beta \mathrm{L}-\mathrm{Kp}$ ) have been reported in several countries and the data from the EARS-NET database show that in 2015 the prevalence of third generation cephalosporin-resistant $\mathrm{Kp}$ isolates was of $57.3 \%$. $^{6}$

Among others, outbreaks caused by AmpC-producing $\mathrm{Kp}$ belonging to sequence types 14 and 26 (ST14 and ST26) and carbapenemase-producing Kp ST258 have been described in Italian NICUs. $^{7-9}$

In this study, we describe an outbreak that occurred in the NICU of Fondazione IRCCS Policlinico San Matteo Hospital in Pavia from January 2013 to September 2013, and the measures that were implemented in the ward to contain and to solve the issue. We collected $97 \mathrm{ES} \beta \mathrm{L}-\mathrm{Kp}$ strains, and we investigated the molecular, genomic, and epidemiologic features to identify the factors that had contributed to their diffusion.

\section{The Event}

Fondazione IRCCS Policlinico San Matteo in Pavia is a 1000-bed hospital located in Northern Italy.

The NICU is divided into four rooms with seven beds each, connected by a corridor. The medical and nursing staff includes 40 people. Approximately 300 patients are admitted annually. The NICU is a regional referral center for neonatal care and about $23 \%$ of the newborns admitted weigh less than $1,500 \mathrm{~g}$. Prematurity, neonatal hypoxia, and hypoxic-ischemic encephalopathy are the most common reasons for admission.

Figure 1 shows the number of isolations of ES $\beta \mathrm{L}-\mathrm{Kp}$ in the NICU during 2013. Between January and March 2013 ES $\beta \mathrm{L}-\mathrm{Kp}$ was isolated from different sources (conjunctival swabs, low and high respiratory tract samples, blood, urine) from 11 patients, representing $14.1 \%$ of the patients admitted $(n=78)$. Comparing this percentage with one of the same period of the previous year $(3 / 76-3.9 \% p=0.003)$ a clear increase was detected, and the possibility of an outbreak was considered.

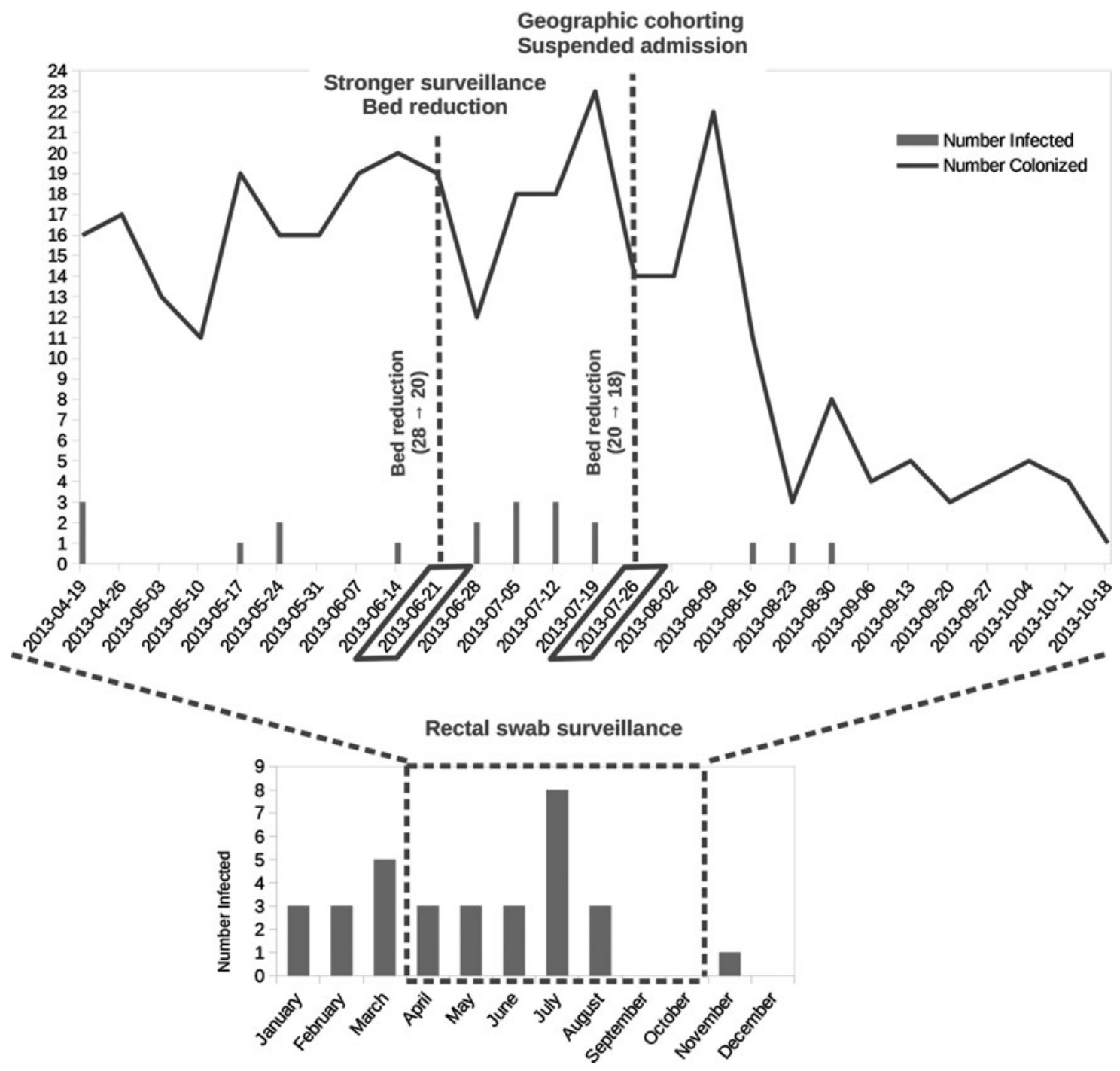

FIG. 1. Above: number of patients of the neonatal intensive care unit colonized (represented with a gray line) and infected (represented with gray bars) by Klebsiella pneumoniae in the period from April to October 2013. Below: number of patients of the neonatal intensive care unit colonized by K. pneumoniae during 2013. 
Starting on April 17th, additional surveillance measures were undertaken: systematic fecal swabs for each patient admitted, increase in the use of disposable devices, daily cleaning, and passive surveillance. These measures did not succeed in reducing ES $\beta \mathrm{L}-\mathrm{Kp}$ presence, and the systematic fecal swab testing highlighted an even stronger pattern of colonization in apparently healthy infants. In the period April-June, 56 patients resulted to be colonized, 8 of which were also infected, out of a total of 75 patients admitted.

These data prompted a second set of stricter control measures implemented on June 19th, including an increased passive surveillance and the reduction of the total beds of the NICU from 28 to 20. This second set of measures was not sufficient to stop the outbreak, as in the following month 21 patients resulted to be colonized, 6 of which were infected, out of a total of 22 patients admitted. On July 24th 2013 the interventions were reinforced again: additional reduction of the total beds of the NICU from 20 to 18 , supplementary training for all healthcare workers, accurate daily cleaning of the surfaces and fomites followed by disinfection, strict geographic cohorting of colonized infants and their nursing staff, and suspension of any new admission. This set of measures allowed to finally reduce the number of newly infected and colonized patients, respectively one and six in August, zero and three in September, zero, and one in October. Since November no additional cases were recorded, while strict surveillance was implemented until the end of the year.

\section{Materials and Methods}

\section{Ethics statement}

The study was designed and conducted in accordance with Helsinki declaration and approved by the Ethics Committee of Fondazione IRCCS Policlinico San Matteo in Pavia, Italy.

\section{Microbiology methods}

All the biological samples collected were sent to the Microbiology and Virology Unit, where they were directly inoculated either on MacConkey agar plates (rectal swabs) or on Columbia agar with 5\% sheep blood plates (conjunctival swabs, blood cultures, cerebrospinal fluid, respiratory samples). Identification and antibiotic susceptibility testing were performed using the Phoenix $100^{\mathrm{TM}}$ system (BD Dickinson and Company, Franklin Lakes, NJ) and interpreted according to EUCAST guidelines. ${ }^{10}$ Phenotypic ES $\beta$ L detection were performed both with the double-disc synergy test ${ }^{11}$ and with ES $\beta \mathrm{L}$ + AmpC Screen kit (Rosco Diagnostica, Taastrup, Denmark). Infection was defined by clinical and laboratory criteria, while colonization was defined by the absence of relevant symptoms.

\section{Molecular microbiology methods}

On the basis of resistance phenotype, specimens type/date of collection, 16 representative ES $\beta \mathrm{L}-\mathrm{Kp}$ isolated on 2013 were selected to perform the phenotypic and molecular characterization. Three isolates previously collected from blood cultures in the same unit in September 2011, August and October 2012 were also included in the study, for a total of 19 strains. Total DNA was extracted from all strains using a QIAamp DNA minikit (Qiagen, Italy) according to manufacturer's instructions.

Polymerase chain reaction (PCR) assays were performed to amplify the genes encoding for ES $\beta \mathrm{Ls}\left(b l a_{\mathrm{CTX}-\mathrm{M}}, b l a_{\mathrm{TEM}}\right.$, $\left.b l a_{\mathrm{OXA}}\right)$ and aac $\left(6^{\prime}\right)-\mathrm{Ib}-\mathrm{cr}$, using primers and conditions previously described. ${ }^{12-15} \mathrm{PCR}$ products were purified using the kit Quantum Prep PCR Kleen Spin Columns (BioRad, Alfred Nobel Drive, Hercules) and subjected to double-strand Sanger sequencing (Macrogen, Inc., Seoul, South Korea).

Production of the $\beta$-lactamases detected with molecular methods was confirmed by isoelettrofocusing (IEF). IEF was performed on polyacrylamide gel containing ampholines with a pH range of 3.0-10.0 (Amersham Biosciences, France). $\beta$ lactamase activities were detected with the chromogen nitrocefin (Oxoid, Basingstoke, Hampshire, England). Activity against CTX substrate of the $\beta$-lactamases bands separated by IEF was assayed by a substrate overlaying procedure, as reported previously. ${ }^{16}$

Conjugal transfer of resistance determinants were performed in liquid medium using the Escherichia coli K12 strain J62 (pro-, his-, trp-, lac-, SmR) and J53 (met-, pro-, rifR) as recipients. The transconjugants were selected on MacConkey agar containing CTX (8 mg/L) plus streptomycin $(1000 \mathrm{mg} / \mathrm{L})$ or rifampicin $(100 \mathrm{mg} / \mathrm{L})$. Plasmids were typed according to their incompatibility group using the PCR replicon-typing scheme as described previously. ${ }^{17}$ The sizes of the plasmids were estimated using the $\mathrm{S} 1$ nuclease pulsed-field gel electrophoresis (PFGE) method. ${ }^{18}$

Clonality of the K. pneumoniae isolates was investigated by PFGE using $X b a$ I restriction enzyme. Fragments were separated on a CHEF DRII system (Bio-Rad, Hercules, CA) at $14^{\circ} \mathrm{C}$ at $6 \mathrm{~V} / \mathrm{cm}$ for $20 \mathrm{hr}$ with an initial pulse time of $0.5 \mathrm{sec}$ and a final pulse time of $30 \mathrm{sec}$. Lambda $48.5 \mathrm{~kb}$ concatamers (New England BioLabs, Beverly, MA) were used as size standard to align the bands. Dendrograms of strain relatedness were created with Fingerprinting II version 3.0 software (Bio-Rad) using UPGMA. The Dice correlation coefficient was used with a $1.5 \%$ position tolerance to analyze the similarities of the banding patterns.

In addition, Multi-locus sequence typing (MLST) was performed based on the sequence analysis of fragments of seven housekeeping genes: rpoB (beta-subunit of RNA polymerase), gapA (glyceraldehyde 3-phosphate dehydrogenase), $m d h$ (malate dehydrogenase), pgi (phosphoglucoseisomerase), phoE (phosphorine E), infB (translation initiation factor 2), and $\operatorname{ton} B$ (periplasmic energy transducer). PCR amplifications were carried out under the following conditions: 35 cycles (denaturation at $94^{\circ} \mathrm{C}$ for $2 \mathrm{~min}$, annealing at $50^{\circ} \mathrm{C}$ for $30 \mathrm{sec}$, and extension at $72^{\circ} \mathrm{C}$ for $30 \mathrm{sec}$ ) preceeded by a 2 min denaturation at $94^{\circ} \mathrm{C}$ and followed by a $5 \mathrm{~min}$ extension at $72^{\circ} \mathrm{C}$. Details of the MLST scheme including amplification and sequencing primers, allele sequences and STs are available on Institute Pasteur's MLST Web site. ${ }^{19}$

\section{Genomic methods}

On the basis of the PFGE results, four strains out of 19 were analyzed using whole genome sequencing: the strain collected in September 2011, one collected in August 2012, and two collected during the outbreak. DNA, previously extracted for molecular analysis, was sequenced on a MiSEQ Illumina machine, after Nextera library construction, to obtain 250 bp paired-ends reads. Reads were quality checked using FastQC (www.bioinformatics.babraham.ac.uk/projects/ fastqc/), and assembled using MIRA 4.0rcl. ${ }^{20}$ Genome assemblies were screened for strict cgMLST $^{21}$ genes and capsular genes using the BIGSdb software. ${ }^{22}$ Finally, genomes 
were scanned for the presence of virulence and antibiotic resistance genes using a BLAST search against the ResFinder ${ }^{23}$ and VirulenceFinder ${ }^{24}$ databases.

\section{Statistical analysis}

Descriptive statistics were produced for demographic features, clinical characteristics, and risk factors of cases. Mean and standard deviation are presented for normally distributed variables, median and interquartile range for non-normally distributed variables, and number and percentages for categorical variables. Groups were compared with parametric or nonparametric tests, according to data distribution for continuous variables, and with Pearson's $\chi^{2}$ test (Fisher exact test where appropriate) for categorical variables. Logistic regression models (univariate analysis only, due to low number of events) were used to explore risk factors associated to infection (vs. colonization). In all cases, two-tailed tests were used. $p$-Value significance cutoff was 0.05 . The Stata version 14.0 software (Stata Corporation, 4905 Lakeway Drive, College Station, TX) was used for statistical analysis.

\section{Results}

\section{Case characteristics}

During the period from January to March, 78 children were hospitalized in the NICU. Among these $11(14.1 \%)$ were infected by ES $\beta$ L-Kp. This high prevalence prompted the declaration of an ES $\beta$ L-Kp ongoing outbreak. During the period April-September passive surveillance was implemented through analysis of rectal swabs. In this period 146 children were hospitalized in the NICU, 81 of them $(55.4 \%)$ were either colonized (66) or infected (15) by ES $\beta$ L-Kp. Figure 1 shows the trend of the colonized patients during the period analyzed, indicating the measures that were progressively implemented until the resolution of the outbreak.

Demographic characteristics, gestational age, birth weight, and several risk factors, such as intubation, placement of central venous catheters and chest tubes are reported in Table 1. Level of prematurity (severe prematurity: gestational age $<30$ weeks), APGAR score at birth and at $10 \mathrm{~min}$, and the length of stay result as significantly associated $(p<0.05)$ with ES $\beta$ L-Kp infection, whereas other demographic and clinical variables have not been identified as risk factors.

\section{Microbiology and molecular results}

From January to September 2013, 97 ES $\beta L-K p$ isolates were obtained from the neonates, 66 of which were from surveillance cultures and 31 from clinical specimens: blood $(n=7)$; high respiratory tract $(n=3)$; lower respiratory tract $(n=1)$; urine $(n=12)$; cerebrospinal fluid specimens $(n=2)$; and conjunctival swabs $(n=6)$.

All 97 isolates showed the same MDR pattern and the phenotypic screening confirmed ES $\beta$ L enzyme production. Sixteen ES $\beta \mathrm{L}-\mathrm{Kp}$ representative strains of the outbreak event, plus three additional ES $\beta$ L-Kp strains obtained in the previous years, were selected for molecular characterization. See Table 2 for patients, isolation, and antibiotic susceptibility characteristics of the 19 isolates selected.

IEF showed four $\beta$-lactamase bands common to $18 / 19$ isolates: pI 8.2, pI 7.5, pI 7.3, and pI of 5.4 generally associated with the expression of CTX-M-type, SHV-type, OXA-type, and TEM-type enzyme, respectively. Only the isolate collected in 2011 showed the presence of a unique $\beta$-lactamase band with $\mathrm{pI}$ 8.2. All the 19 isolates presented a band with hydrolytic activity on CTX $(1 \mu \mathrm{g} / \mathrm{mL})$ at pI of 8.2 by overlay assay, related to the production of CTX-M-type enzyme.

PCR and sequencing showed that all 19 isolates tested carried $b l a_{\mathrm{CTX}-\mathrm{M}-15}$ gene and all, except for the isolate of

Table 1. Demographic Features, Clinical Characteristics, and Risk Factors of Patients: Comparison Between Colonized $(N=66)$ and Infected Subjects $(N=26)$

\begin{tabular}{llcccc}
\hline Variable & $\begin{array}{c}\text { Descriptive } \\
\text { statistic }\end{array}$ & $\begin{array}{c}\text { Colonized } \\
(\mathrm{n}=66)\end{array}$ & $\begin{array}{c}\text { Infected } \\
(\mathrm{n}=26)\end{array}$ & $\begin{array}{c}\text { OR } \\
\text { O5\% }\end{array}$ & $\begin{array}{c}\mathrm{p} \text {-Value } \\
\text { CI }\end{array}$ \\
\hline Gendivariate)
\end{tabular}

${ }^{\mathrm{a}}$ per $100 \mathrm{~g}$.

${ }^{\mathrm{b}}$ One missing data.

${ }^{\mathrm{c}}$ Two missing data.

OR, odds ratio; CI, confidence interval; IQR, interquartile range. 


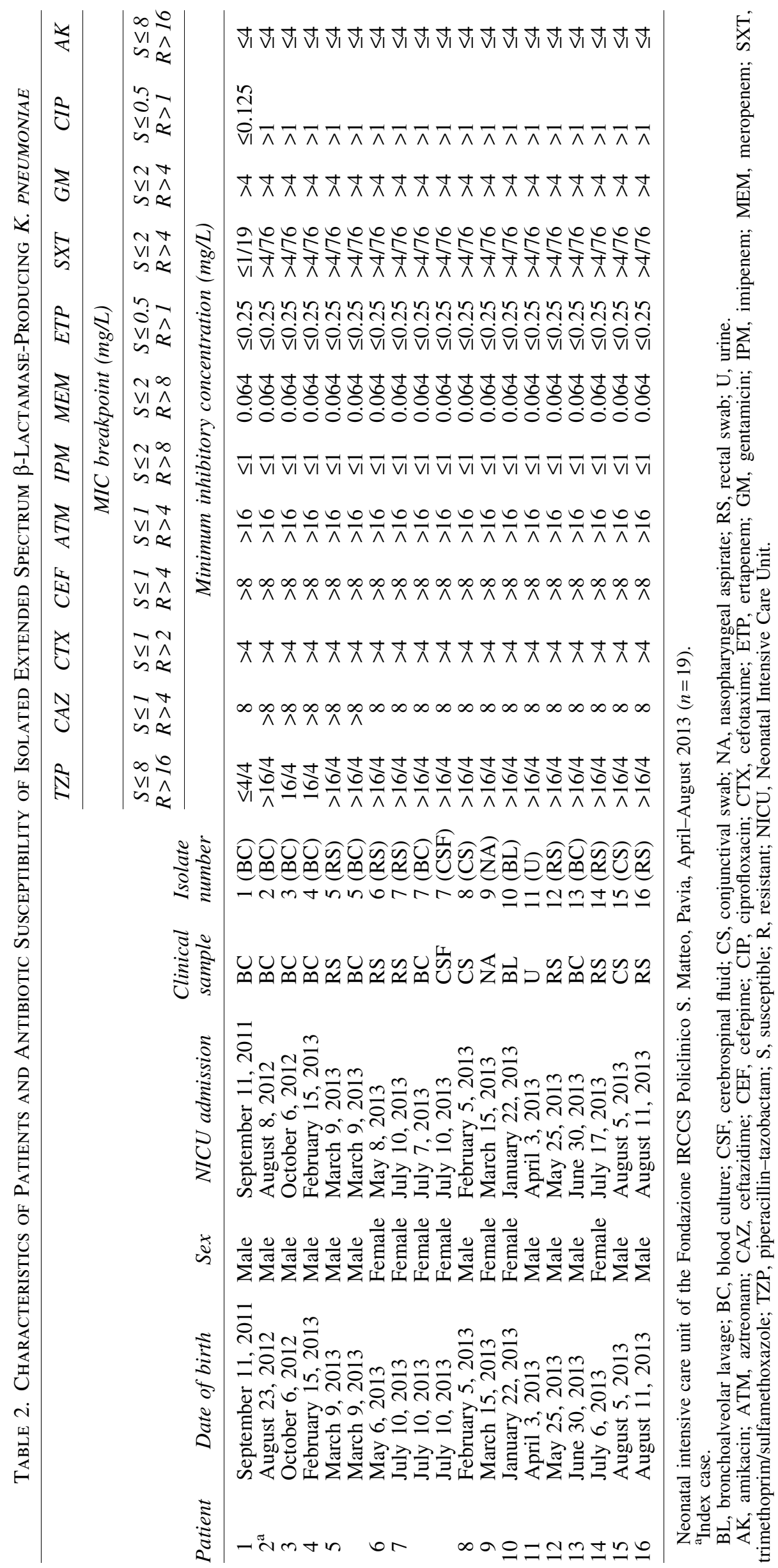


Table 3. Molecular Characteristics of Extended Spectrum $\beta$-Lactamase-Producing K. PNeUmonia (N=19)

\begin{tabular}{|c|c|c|c|c|c|}
\hline Isolate & $I E F$ & $\begin{array}{c}\text { Overlay } \\
\text { assay }\end{array}$ & Resistance genes (polymerase chain reaction) & $M L S T$ & $\begin{array}{l}\text { PFGE } \\
\text { clone }\end{array}$ \\
\hline $1(\mathrm{BC})$ & pI 8.2 & pI 8.2 & blaCTX-M- & ST14 & $\mathrm{B}$ \\
\hline $2(\mathrm{BC})$ & pI 8.2, pI 7.5, pI 7.3, pI 5.4 & pI 8.2 & blaCTX-M-15, blaOXA-1, blaTEM-206, aac(6')-Ib-cr & ST307 & A \\
\hline $3(\mathrm{BC})$ & pI 8.2, pI 7.5, pI 7.3, pI 5.4 & pI 8.2 & blaCTX-M-15, blaOXA-1, blaTEM-206, aac(6')-Ib-cr & ST307 & A \\
\hline $4(\mathrm{BC})$ & pI 8.2, pI 7.5, pI 7.3, pI 5.4 & pI 8.2 & blaCTX-M-15, blaOXA-1, blaTEM-206, aac(6')-Ib-cr & ST307 & A \\
\hline $5(\mathrm{RS})$ & pI 8.2, pI 7.5, pI 7.3, pI 5.4 & pI 8.2 & blaCTX-M-15, blaOXA-1, blaTEM-206, aac(6')-Ib-cr & ST307 & A \\
\hline $5(\mathrm{BC})$ & pI 8.2, pI 7.5, pI 7.3, pI 5.4 & pI 8.2 & blaCTX-M-15, blaOXA-1, blaTEM-206, aac(6')-Ib-cr & ST307 & A \\
\hline $6(\mathrm{RS})$ & pI 8.2, pI 7.5, pI 7.3, pI 5.4 & pI 8.2 & blaCTX-M-15, blaOXA-1, blaTEM-206, aac(6')-Ib-cr & ST307 & A \\
\hline 7 (RS) & pI 8.2, pI 7.5, pI 7.3, pI 5.4 & pI 8.2 & blaCTX-M-15, blaOXA-1, blaTEM-206, aac(6')-Ib-cr & ST307 & A \\
\hline $7(\mathrm{BC})$ & pI 8.2, pI 7.5, pI 7.3, pI 5.4 & pI 8.2 & blaCTX-M-15, blaOXA-1, blaTEM-206, aac(6')-Ib-cr & ST307 & A \\
\hline 7 (CSF) & pI 8.2, pI 7.5, pI 7.3, pI 5.4 & pI 8 & blaCTX-M-15, blaOXA-1, blaTEM-206, aac(6')-Ib-cr & ST307 & A \\
\hline $8(\mathrm{CS})$ & 5.4 & & blaCTX-M-15, blaOXA-1, blaTE & $\mathrm{S}]$ & A \\
\hline 9 (NA) & pI 8.2, pI 7.5, pI 7.3, pI 5.4 & pI 8.2 & blaCTX-M-15, blaOXA-1, blaTEM-206, aac (6')-Ib-cr & ST307 & A \\
\hline $10(\mathrm{BL})$ & pI 8.2, pI 7.5, pI 7.3, pI 5.4 & pI 8.2 & blaCTX-M-15, blaOXA-1, blaTEM-206, aac(6')-Ib-cr & ST307 & A \\
\hline $11(\mathrm{U})$ & pI 8.2, pI 7.5, pI 7.3, pI 5.4 & pI 8.2 & blaCTX-M-15, blaOXA-1, blaTEM-206, aac(6')-Ib-cr & ST307 & A \\
\hline $12(\mathrm{RS})$ & pI 8.2, pI 7.5, pI 7.3, pI 5.4 & pI 8.2 & blaCTX-M-15, blaOXA-1, blaTEM-206, aac(6')-Ib-cr & ST307 & A \\
\hline $13(\mathrm{BC})$ & pI 8.2, pI 7.5, pI 7.3, pI 5.4 & pI 8.2 & blaCTX-M-15, blaOXA-1, blaTEM-206, aac(6')-Ib-cr & ST307 & A \\
\hline $14(\mathrm{RS})$ & pI 8.2, pI 7.5, pI 7.3, pI 5.4 & pI 8.2 & blaCTX-M-15, blaOXA-1, blaTEM-206, aac(6')-Ib-cr & ST307 & A \\
\hline $15(\mathrm{CS})$ & pI 8.2, pI 7.5, pI 7.3, pI 5.4 & pI 8.2 & blaCTX-M-15, blaOXA-1, blaTEM-206, aac(6')-Ib-cr & ST307 & A \\
\hline $16(\mathrm{RS})$ & pI 8.2, pI 7.5, pI 7.3, pI 5.4 & pI 8.2 & blaCTX-M-15, blaOXA-1, blaTEM-206, aac(6')-Ib-cr & ST307 & A \\
\hline
\end{tabular}

IEF, isoelettrofocusing; MLST, multi-locus sequence typing; PFGE, pulsed-field gel electrophoresis.

2011, additionally carried $b l a_{\mathrm{OXA}-1}, b l a_{\mathrm{TEM}-206}$, and aac(6')Ib-cr gene (Table 3).

All the isolates analyzed were able to perform conjugation. The transfer of the ES $\beta \mathrm{L}$ determinant was observed at a frequency of $\sim 10^{-3}$ transconjugants per recipient. Plasmid analysis indicated that all but the 2011 isolate had acquired a plasmid of about $200 \mathrm{~kb}$ belonging to the IncF incompatibility group.

PFGE clustered 18 of the 19 isolates into a clonal type named A, which exhibited two subtypes, A1 and A2. The isolate collected in 2011 was the only one belonging to a different clonal type, named $\mathrm{B}^{25}$ MLST assigned the 18 isolates (collected from 2012 to 2013) of the dominant clonal type A to sequence type ST307, while the isolate collected in 2011 was assigned to ST14. The temporal distribution of ES $\beta \mathrm{L}-$ producers and their respective clonal type are shown in Fig. 2.

Four representative strains were selected for Illumina sequencing. They are 1 (BC) (the isolate collected in 2011), 2 (BC) (the isolate collected in August 2012), 7 (BC), and 13 (BC) (two isolates collected in 2013). In-silico MLST confirmed the amplicon sequencing results (i.e., ST307 for the three clone A isolates and ST14 for the 2011 strain). Furthermore, the three sequenced clone A isolates were assigned to the KN2 capsular type through the presence of the wzi-173 capsular antigen allele, while the isolate collected in 2011 turned out to have capsular type K2 (wzi-2).

Out of 634 strict cgMLST genes, 605 were found in all four genomes. The three genomes of clone A shared the same allele for all 605 genes, while Kp1734 differed for 506 alleles from the other three (for further details, see Supplementary Table S1; Supplementary Data are available online at www.liebertpub.com/mdr). BLAST-search against the Virulence Finder database allowed to characterize the genomes further (Table 4). In detail, all four isolates presented the genes encoding the type 3 fimbrial proteins ( $m r k A B D F H I J)$ and gene iutA (coding for a siderophore). Isolate Kp1734 was characterized by the presence of genes $k f u A, k f u B$, and $k f u C$ (responsible for iron acquisition), which were not detected in the three ST307 genomes.

As regards antibiotic resistance, determinants for resis-

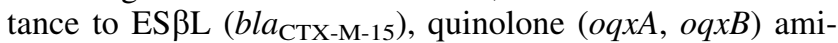
noglycoside ( $a a c(3)-I I a)$, and fosfomycin (fosA) resistance genes were identified in all isolates. In addition, genomes of clone A carried an array of genes that are not present in the genome of clone B. This includes $\operatorname{str} A$ and $\operatorname{str} B$ (aminoglycoside), $b l a_{\text {SHV-28 }}, b l a_{\text {TEM-206 }}, b_{\text {OXA-1 }}$ (beta-lactams), $a a c\left(6^{\prime}\right) I b-c r$ (fluoroquinolone and aminoglycoside resistance), catB3 (phenicol), qnrB66 (quinolone), sul2 (sulfonamide), tet(A) (tetracycline), and $d f r A 14$ (trimethoprim) (see Table 4 for further details).

The sequences of the four genomes are available with the following EBI accession numbers: 1 (BC) — as PV1BC - under ERS1864686, 2 (BC)—as PV2BC-under ERS1864687, 7 (BC) - as PV7BC - under ERS1864688, and 13 (BC) - as PV13BC-under ERS1864689.

\section{Discussion}

Patients admitted to NICUs represent a population at high risk of infection with ES $\beta \mathrm{L}-\mathrm{Kp}$ strains, an important cause of morbidity and mortality due to the limited treatment options. Indeed these strains exhibit coexistence of ES $\beta L$ production with resistance to quinolones, aminoglycosides, and trimethoprim-sulfamethoxazole, and have thus become a sanitary threat worldwide. ${ }^{26}$

In this study, we describe an outbreak of ES $\beta$ L-Kp, which occurred during the period January-September 2013 in the NICU of the Fondazione IRCCS Policlinico San Matteo Hospital in Pavia, and that affected 92 infants, 26 infected and 66 colonized. A total of $97 \mathrm{ES} \beta \mathrm{L}-\mathrm{Kp}$ strains were isolated during the outbreak period: 16 of them, together with 3 strains that had been isolated previously, were subjected to molecular characterization. A clonal pattern was observed among 18/19 ES $\beta$ L-Kp isolates: not just the 16 outbreak 

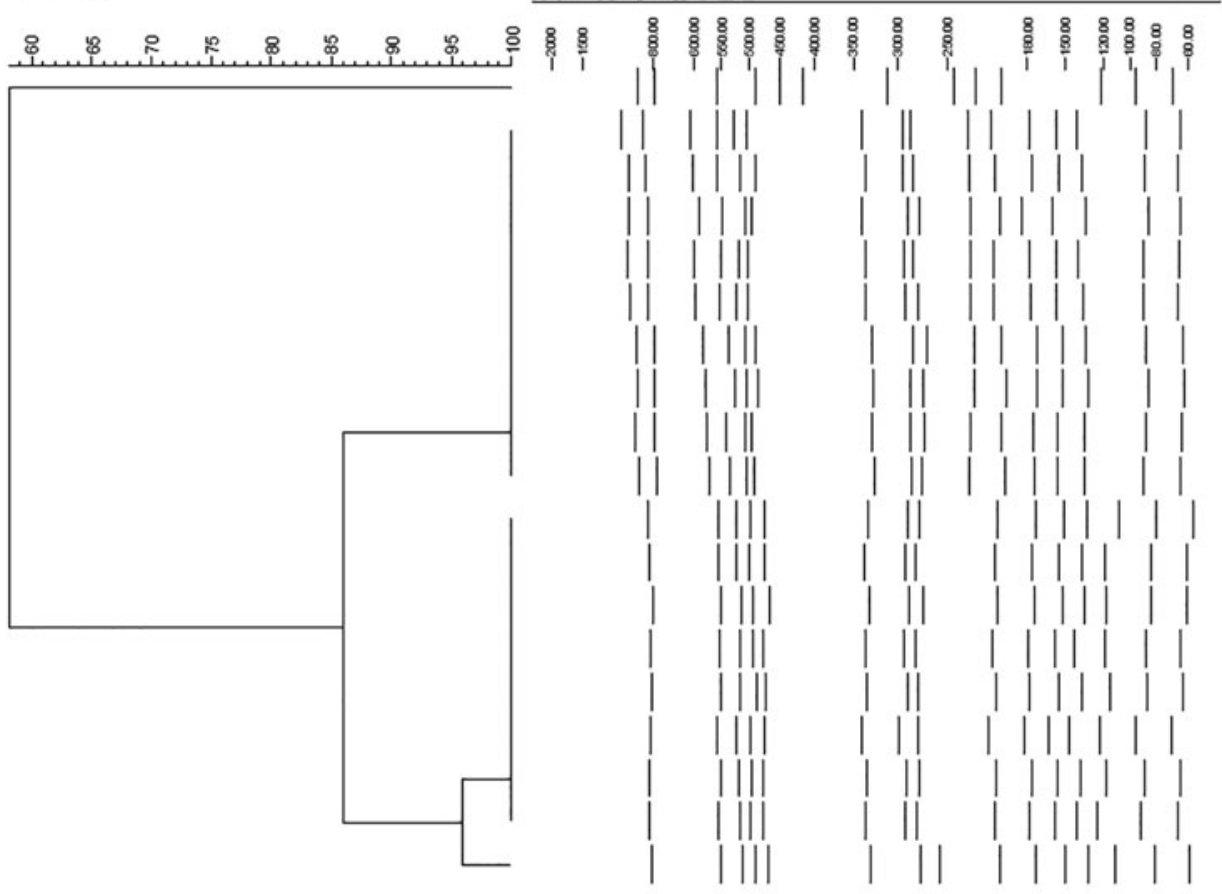

\begin{tabular}{lll} 
PFGE clone & Isolate & Isolation Date \\
\hline $\mathrm{B}$ & $1(\mathrm{BC})$ & $11 / 09 / 2011$ \\
\hline $\mathrm{A}$ & $3(\mathrm{BC})$ & $06 / 10 / 2012$ \\
\hline $\mathrm{A}$ & $4(\mathrm{BC})$ & $15 / 02 / 2013$ \\
\hline $\mathrm{A}$ & $5(\mathrm{BC})$ & $09 / 03 / 2013$ \\
\hline $\mathrm{A}$ & $5(\mathrm{RS})$ & $09 / 03 / 2013$ \\
\hline $\mathrm{A}$ & $6(\mathrm{RS})$ & $08 / 05 / 2013$ \\
\hline $\mathrm{A}$ & $7(\mathrm{RS})$ & $10 / 07 / 2013$ \\
\hline $\mathrm{A}$ & $7(\mathrm{BC})$ & $10 / 07 / 2013$ \\
\hline $\mathrm{A}$ & $7(\mathrm{CSF})$ & $10 / 07 / 2013$ \\
\hline $\mathrm{A} 1$ & $2(\mathrm{BC})$ & $23 / 08 / 2012$ \\
\hline $\mathrm{A} 1$ & $12(\mathrm{RS})$ & $25 / 05 / 2013$ \\
\hline $\mathrm{A} 1$ & $10(\mathrm{BL})$ & $22 / 01 / 2013$ \\
\hline $\mathrm{A} 1$ & $11(\mathrm{U})$ & $03 / 04 / 2013$ \\
\hline $\mathrm{A} 1$ & $15(\mathrm{CS})$ & $05 / 08 / 2013$ \\
\hline $\mathrm{A} 1$ & $9(\mathrm{NA})$ & $15 / 03 / 2013$ \\
\hline $\mathrm{A} 1$ & $14(\mathrm{RS})$ & $17 / 07 / 2013$ \\
\hline $\mathrm{A} 1$ & $16(\mathrm{RS})$ & $11 / 08 / 2013$ \\
\hline $\mathrm{A} 2$ & $8(\mathrm{CS})$ & $05 / 02 / 2013$ \\
\hline & $13(\mathrm{BC})$ & $30 / 06 / 2013$ \\
\hline
\end{tabular}

FIG. 2. Cluster analysis of the 19 ES $\beta L s-p r o d u c i n g ~ K$. pneumoniae pulsed-field gel electrophoresis profiles. Lambda $48.5 \mathrm{~kb}$ concatamers (New England BioLabs, Beverly, MA) were used as size standard to align the bands. Date of isolation is also included. The scale bar at the top (left) indicates similarity coefficient (\%). ES $\beta$ Ls, extended spectrum $\beta$-lactamases.

Table 4. Presence of Genes (and Alleles) Related to Antimicrobial Resistance and Virulence in the Four SeQuenced Genomes

\begin{tabular}{|c|c|c|c|c|}
\hline Associated phenotypelfunction & $13(B C)$ & $7(B C)$ & $2(B C)$ & $1(B C)$ \\
\hline Aminoglycoside resistance & strA & strA & strA & $/ /$ \\
\hline Aminoglycoside resistance & $\operatorname{str} B$ & strB & $\operatorname{str} B$ & $/ /$ \\
\hline Aminoglycoside resistance & aac(3)-IIa & aac(3)-IIa & aac(3)-IIa & aac(3)-IIa \\
\hline Beta-lactam resistance & $b l a_{\mathrm{SHV}-28}$ & $b l a_{\mathrm{SHV}-28}$ & $b l a_{\mathrm{SHV}-28}$ & $/ /$ \\
\hline Beta-lactam resistance & bla $a_{\text {TEM-206 }}$ & bla $a_{\mathrm{TEM}-206}$ & bla $a_{\mathrm{TEM}-206}$ & $/ /$ \\
\hline Beta-lactam resistance & bla $_{\mathrm{CTX}-\mathrm{M}-15}$ & $b^{b} a_{\mathrm{CTX}-\mathrm{M}-15}$ & $b_{l a} a_{\mathrm{CTX}-\mathrm{M}-15}$ & bla $a_{\mathrm{CTX}-\mathrm{M}-15}$ \\
\hline Beta-lactam resistance & bla $a_{\mathrm{OXA}-1}$ & $b l a_{\mathrm{OXA}-1}$ & $b l a_{\mathrm{OXA}-1}$ & $/ /$ \\
\hline Beta-lactam resistance & $/ /$ & $/ /$ & $/ /$ & $b l a_{\mathrm{TEM}-1 \mathrm{~B}}$ \\
\hline Beta-lactam resistance & $/ /$ & $/ /$ & $/ /$ & bla $_{\mathrm{SCO}-1}$ \\
\hline Fluoroquinolone and aminoglycoside resistance & $\operatorname{aac}\left(6^{\prime}\right) \mathrm{Ib}-\mathrm{cr}$ & $\mathrm{aac}\left(6^{\prime}\right) \mathrm{Ib}-\mathrm{cr}$ & $\operatorname{aac}\left(6^{\prime}\right) \mathrm{Ib}-\mathrm{cr}$ & $/ /$ \\
\hline Fosfomycin resistance & fos $A$ & fos $A$ & fos $A$ & fos $A$ \\
\hline Phenicol resistance & catB3 & catB3 & catB3 & $/ /$ \\
\hline Quinolone resistance & $o q x B$ & $o q x B$ & $o q x B$ & $o q x B$ \\
\hline Quinolone resistance & $o q x A$ & oqxA & oqxA & $o q x A$ \\
\hline Quinolone resistance & qnrB66 & qnrB66 & qnrB66 & $/ /$ \\
\hline Sulfonamide resistance & sul2 & sul2 & sul2 & $/ /$ \\
\hline Tetracycline resistance & $\operatorname{tet}(A)$ & $\operatorname{tet}(A)$ & $\operatorname{tet}(A)$ & $/ /$ \\
\hline Trimethoprim resistance & dfrA 14 & dfrA14 & dfrA14 & $/ /$ \\
\hline Siderophore & iutA & iutA & iutA & iutA \\
\hline Siderophore & $/ /$ & $/ /$ & $/ /$ & $k f u A$ \\
\hline Siderophore & // & $/ /$ & $/ /$ & $k f u B$ \\
\hline Siderophore & $/ /$ & $/ /$ & $/ /$ & $k f u C$ \\
\hline Fimbriae & $m r k A$ & $m r k A$ & $m r k A$ & $m r k A$ \\
\hline Fimbriae & $m r k B$ & $m r k B$ & $m r k B$ & $m r k B$ \\
\hline Fimbriae & $m r k C$ & $m r k C$ & $m r k C 7$ & $m r k C$ \\
\hline Fimbriae & $m r k D$ & $m r k D$ & $m r k D$ & $m r k D$ \\
\hline Fimbriae & $m r k F$ & $m r k F$ & $m r k F$ & $m r k F$ \\
\hline Fimbriae & $m r k H$ & $m r k H$ & $m r k H$ & $m r k H$ \\
\hline Fimbriae & $m r k I$ & $m r k I$ & $m r k I$ & $m r k I$ \\
\hline Fimbriae & $m r k J$ & $m r k J$ & $m r k J$ & $m r k J$ \\
\hline Capsular antigen & $w z i-173$ & $w z i-173$ & $w z i-173$ & $w z i-2$ \\
\hline
\end{tabular}

//, not detected. 
strains, but also the two strains collected in 2012 turned out to belong to a single PFGE clone and to ST307. This result clearly indicates that the outbreak strain had been present in the NICU for at least 6 months before the start of the outbreak.

These 18 strains belonging to ST307 carried $b l a_{\mathrm{CTX}-\mathrm{M}-15}$ gene, $b l a_{\mathrm{OXA}-1}, b l a_{\mathrm{TEM}-206}$, and aac $\left(6^{\prime}\right)$-Ib-cr genes. To our knowledge, this is the first report of $K$. pneumoniae carrying $b l a_{\text {TEM-206 }}$ in clinical isolates. It was previously found in $E$. coli isolates from farmed chickens in China and in $E$. coli isolates from pigs in Germany. ${ }^{27,28}$ Genomics was used to further characterize four isolates $(1[\mathrm{BC}], 2[\mathrm{BC}], 7[\mathrm{BC}]$, and $13[\mathrm{BC}])$. The results confirmed the unrelatedness of the strain isolated in 2011 with the other three, which present a different cgMLST profile and carry different determinants for antimicrobial resistance and virulence. Furthermore, genomic characterization confirmed the clonality of the strains of 2013 and that isolated in 2012.

Many ES $\beta$ L-Kp outbreaks have been reported worldwide, due to the capability of this organism to survive in nosocomial environments, ${ }^{29}$ including NICUs. Strategies to control outbreaks of MDR bacteria colonization/infection in the NICUs may include strict implementation of hands hygiene measures, isolating patients, screening healthcare workers, and performing admission and periodic surveillance cultures. Nevertheless, the standard interventions of infection control within the NICU were in this case not sufficient to terminate the outbreak. Only suspension of admission and cohorting colonized/infected from uncolonized/uninfected newborns into two separate rooms with dedicated staff reduced and later stopped the diffusion of ES $\beta \mathrm{L}-\mathrm{Kp}$, as testified by the complete absence of new cases (Fig. 1) during the following months.

Finally, this study reports an ST307 K. pneumoniae clone which, carrying resistance and virulence genes, imposed significant therapeutic limitations on the treatment of hospital- and community-acquired infections and could compete with the worldwide ST258 clone, ${ }^{30,31}$ although larger studies are needed to determine the real epidemiological importance. Nevertheless, the presence of new resistance genes reinforces the necessity for permanent surveillance programs. Local epidemiologic studies, specific for single countries, regions, or even single hospitals, are needed to help physicians in managing their patients in daily practice, for microbiologists to detect more accurately the resistance patterns of pathogens circulating within their hospital, and for both to implement more efficient systems of infection control to prevent the spread of multidrug-resistant $K$. pneumoniae infections.

\section{Acknowledgments}

This work was supported by Ricerca Corrente 2013 funding from Fondazione IRCCS Policlinico San Matteo to P.C. The authors would like to thank Carolina Ferrari, Piero Marone, and Laura Pagani for their help in the results interpretation, Marina Lanave for her help in designing the strategy adopted for outbreak containment, and Brenda Bronfman for grammar review.

\section{Disclosure Statement}

No competing financial interests exist.

\section{References}

1. Berezin, E.N., F. Solórzano; Latin America Working Group on Bacterial Resistance. 2014. Gram-negative infections in pediatric and neonatal intensive care units of Latin America. J. Infect. Dev. Ctries. 8:942-953.

2. Cipolla, D., M. Giuffrè, C. Mammina, and G. Corsello. 2011. Prevention of nosocomial infections and surveillance of emerging resistances in NICU. J. Matern. Fetal. Neonatal Med. 24 Suppl 1:23-26.

3. Sohn, A.H., D.O. Garrett, R.L. Sinkowitz-Cochran, L.A. Grohskopf, G.L. Levine, B.H. Stover, J.D. Siegel, and W.R. Jarvis. 2001. Prevalence of nosocomial infections in neonatal intensive care unit patients: results from the first national point-prevalence survey. J. Pediatr. 139:821-827.

4. Onori, R., S. Gaiarsa,, F. Comandatore, S. Pongolini, S. Brisse, A. Colombo, G. Cassani, P. Marone, P. Grossi, G. Minoja, C. Bandi, D. Sassera, and A. Toniolo. 2015. Tracking nosocomial Klebsiella pneumoniae infections and outbreaks by whole-genome analysis: small-scale Italian Scenario within a Single Hospital. J. Clin. Microbiol. 53:2861-2868.

5. Mavroidi, A., A. Liakopoulos, A. Gounaris, M. Goudesidou, K. Gaitana, V. Miriagou, and E. Petinaki. 2014. Successful control of a neonatal outbreak caused mainly by ST20 multidrug-resistant SHV-5-producing Klebsiella pneumoniae, Greece. BMC Pediatr. 14:105.

6. European Centre for Disease Prevention and Control (ECDC). 2016. European Antimicrobial Resistance Surveillance Network (EARS-Net) database. European Centre for Disease Prevention and Control (ECDC). Available at http://ecdc.europa.eu/en/activities/surveillance/EARSNet/ database/Pages/database.aspx.

7. Nordberg, V., A.Q. Peralta, T. Galindo, A. Turlej-Rogacka, A. Iversen, C.G. Giske, and L. Navér. 2013. High proportion of intestinal colonization with successful epidemic clones of ESBL-producing Enterobacteriaceae in a Neonatal Intensive Care Unit in Ecuador. PLoS One 8:e76597.

8. Arena, F., T. Giani, E. Becucci, V. Conte, G. Zanelli, M.M. D'Andrea, G. Buonocore, F. Bagnoli, A. Zanchi, F. Montagnani, and G.M. Rossolini. 2013. Large oligoclonal outbreak due to Klebsiella pneumoniae ST14 and ST26 producing the FOX-7 AmpC -Lactamase in a Neonatal Intensive Care Unit. J. Clin. Microbiol. 51:4067-4072.

9. Giuffrè, M., C. Bonura, D.M. Geraci, L. Saporito, R. Catalano, S. Di Noto, F. Nociforo, G. Corsello, and C. Mammina. 2013. Successful control of an outbreak of colonization by Klebsiella pneumoniae carbapenemase-producing $K$. pneumoniae sequence type 258 in a neonatal intensive care unit, Italy. J. Hosp. Infect. 85:233-236.

10. The European Committee on Antimicrobial Susceptibility Testing. 2014. Breakpoint Tables for Interpretation of MICs and Zone Diameters. Version 4.0. The European Committee on Antimicrobial Susceptibility Testing Available at www.eucast.org

11. Jarlier, V., M.H. Nicolas, G. Fournier, and A. Philippon. 1988. Extended broad-spectrum beta-lactamases conferring transferable resistance to newer beta-lactam agents in Enterobacteriaceae: hospital prevalence and susceptibility patterns. Rev. Infect. Dis. 10:867-878.

12. Mugnaioli, C., F. Luzzaro, F. De Luca, G. Brigante, G. Amicosante, and G.M. Rossolini. 2005. Dissemination of CTX-M-type extended-spectrum beta-lactamase genes to unusual hosts. J. Clin. Microbiol. 43:4183-4185.

13. Pallecchi, L., M. Malossi, A. Mantella, E. Gotuzzo, C. Trigoso, A. Bartoloni, F. Paradisi, G. Kronvall, and G.M. 
Rossolini. 2004. Detection of CTX-M-type beta-lactamase genes in fecal Escherichia coli isolates from healthy children in Bolivia and Peru. Antimicrob. Agents Chemother. 48: 4556-4561.

14. Coque, T.M., A. Novais, A. Carattoli, L. Poirel, J. Pitout, L. Peixe, F. Baquero, R. Cantón, and P. Nordmann. 2008. Dissemination of clonally related Escherichia coli strains expressing extended-spectrum beta-lactamase CTX-M-15. Emerg. Infect. Dis. 14:195-200.

15. Robicsek, A., J. Strahilevitz, G.A. Jacoby, M. Macielag, D. Abbanat, C.H. Park, K. Bush, and D.C. Hooper. 2006. Fluoroquinolone-modifying enzyme: a new adaptation of a common aminoglycoside acetyltransferase. Nat. Med. 12:83-88.

16. Pagani, L., R. Migliavacca, L. Pallecchi, C. Matti, E. Giacobone, G. Amicosante, E. Romero, and GM. Rossolini. 2002. Emerging extended-spectrum -lactamases in proteus mirabilis. J. Clin. Microbiol. 40:1549-1552.

17. Carattoli, A., V. Miriagou, A. Bertini, A. Loli, C. Colinon, L. Villa, J.M. Whichard, and G.M. Rossolini. 2006. Replicon typing of plasmids encoding resistance to newer beta-lactams. Emerg. Infect. Dis. 12:1145-1148.

18. Barton, B.M., G.P. Harding, and A.J. Zuccarelli. 1995. A general method for detecting and sizing large plasmids. Anal. Biochem. 226:235-240.

19. Klebsiella pneumoniae MLST Database. 2014. Institut Pasteur, Paris. Available at www.pasteur.fr/recherche/ genopole/PF8/mlst/Kpneumoniae.html

20. Chevreux, B., T. Wetter, and S. Suhai. 1999. Genome sequence assembly using trace signals and additional sequence information. Comput. Sci. Biol. 99:, 45-56.

21. Bialek-Davenet, S., A. Criscuolo, F. Ailloud, V. Passet, L. Jones, A.S. Delannoy-Vieillard, B. Garin, S. Le Hello, G. Arlet, M.-H. Nicolas-Chanoine, D. Decré, D., and S. Brisse. 2014. Genomic definition of hypervirulent and multidrugresistant Klebsiella pneumoniae clonal groups. Emerg. Infect. Dis. 20:1812-1820.

22. Jolley, K.A., and M.C.J. Maiden. 2010. BIGSdb: scalable analysis of bacterial genome variation at the population level. BMC Bioinformatics 11:595.

23. Zankari, E., H. Hasman, S. Cosentino, M. Vestergaard, S. Rasmussen, O. Lund, F.M. Aarestrup, and M.V. Larsen. 2012. Identification of acquired antimicrobial resistance genes. J. Antimicrob. Chemother. 67, 2640-2644.

24. Joensen, K.G., F. Scheutz, O. Lund, H. Hasman, R.S. Kaas, E.M. Nielsen, and F.M. Aarestrup. 2014. Real-time wholegenome sequencing for routine typing, surveillance, and outbreak detection of verotoxigenic Escherichia coli. J. Clin. Microbiol. 52:1501-1510.
25. Tenover, F.C., R.D. Arbeit, R.V. Goering, P.A. Mickelsen, B.E. Murray, D.H. Persing, and B. Swaminathan. 1995. Interpreting chromosomal DNA restriction patterns produced by pulsed-field gel electrophoresis: criteria for bacterial strain typing. J. Clin. Microbiol. 33:2233-2239.

26. Raei, F., F. Eftekhar, and M.M. Feizabadi. 2014. Prevalence of quinolone resistance among extended-spectrum $\beta$-lactamase producing uropathogenic Klebsiella pneumoniae. Jundishapur J. Microbiol. 7:e10887.

27. Li, L., B. Wang, S. Feng, J. Li, C. Wu, Y. Wang, X. Ruan, and M. Zeng. 2014. Prevalence and characteristics of extended-spectrum $\beta$-lactamase and plasmid-mediated fluoroquinolone resistance genes in Escherichia coli isolated from chickens in Anhui province, China. PLoS One 9:e104356.

28. Von Salviati, C., A. Friese, N. Roschanski, H. Laube, B. Guerra, A. Käsbohrer, L. Kreienbrock, and U. Roesler.2014. Extended-spectrum beta-lactamases (ESBL)/AmpC betalactamases-producing Escherichia coli in German fattening pig farms: a longitudinal study. Berl. Munch. Tierarztl. Wochenschr. 127:412-419.

29. Banerjee, T., A. Bhattacharjee, S. Upadhyay, S. Mishra, K. Tiwari, S. Anupurba, M.R. Sen, S. Basu, and A. Kumar. 2016. Long-term outbreak of Klebsiella pneumoniae \& third generation cephalosporin use in a neonatal intensive care unit in north India. Indian J. Med. Res. 144:622-629.

30. Dhar, S., E.T. Martin, P.R. Lephart, J.P. McRoberts, T. Chopra, T.T. Burger, R. Tal-Jasper, K. Hayakawa, H. OferFriedman, T. Lazarovitch, R. Zaidenstein, F. Perez, R.A. Bonomo, K.S. Kaye, and D. Marchaim. 2016. Risk factors and outcomes for carbapenem-resistant Klebsiella pneumoniae isolation, stratified by its multilocus sequence typing: ST258 versus non-ST258. Open Forum Infect Dis 3:ofv213.

31. Lee, C.-R., J.H. Lee, K.S. Park, Y.B. Kim, B.C. Jeong, and S.H. Lee. 2016. Global dissemination of carbapenemaseproducing Klebsiella pneumoniae: epidemiology, genetic context, treatment options, and detection methods. Front. Microbiol. 7:895.

Address correspondence to: Marta Corbella, SC U.O.C. Microbiologia e Virologia Fondazione IRCCS Policlinico San Matteo Viale Taramelli 5 Pavia 27100 Italy

E-mail: m.corbella@smatteo.pv.it 